\title{
ORDER AND DIMENSION
}

\author{
RICHARD M. COHN ${ }^{1}$
}

\begin{abstract}
It will be shown that the conjectured Jacobi bound for the order of differential systems cannot be valid if a very natural conjecture concerning the differential dimension of such systems is false.
\end{abstract}

1. Summary. It will be shown that the conjectured Jacobi bound for the order of differential systems cannot be valid if a very natural conjecture concerning the differential dimension of such systems is false. It is indeed obvious that in certain cases in which the Jacobi bound is $-\infty$ (so that it is considered to hold only if the system has no component of finite order) the Jacobi bound conjecture must be false if the conjecture concerning dimension is false. It will be shown that if the conjecture concerning dimension is false, then there are even cases in which the Jacobi bound is finite but invalid.

2. Notation. $K$ is throughout an ordinary differential field of characteristic 0 . Superscripts denote differentiation.

3. Introduction. Let $S$ be a system of $k$ differential polynomials in $n>k$ differential indeterminates with coefficients in $K$. A very natural conjecture [8, Appendix, paragraph 10] is that if the manifold of $S$ is not empty, then every component of the manifold is of positive differential dimension. This conjecture will be referred to as the dimension conjecture. If the dimension conjecture is false, then the strong Jacobi bound for order (definition below) must be invalid. For let $S$ be a system which violates the dimension conjecture, and let $T$ be obtained from $S$ by adjoining the 0 polynomial $n-k$ times. Then $T$ is a system of $n$ differential polynomials in $n$ indeterminates. The strong Jacobi bound for $T$ is $-\infty$, but $T$ has a component of differential dimension 0 . The relation between the dimension conjecture and the conjecture that the strong Jacobi bound is valid when it is $-\infty$ has been investigated in detail by Joseph Tomasovic $[9, \S 6]$ for partial as well as ordinary differential equations. He proves the equivalence of these conjectures as well as other results.

We are left with the question of the relation between the dimension conjecture and the strong Jacobi bound in the case that this bound is finite. It is the purpose of this

Received by the editors December 11, 1981

1980 Mathematics Subject Classification. Primary 12H05

Key words and phrases. Jacobi bound, differential dimension, order, dimension conjecture, Ritt bound.

'The author is grateful to the Rutgers University Faculty Academic Study Plan for leave which made possible the writing of this paper. 
note to show that if the dimension conjecture is false, then the strong Jacobi bound, and also the weak Jacobi bound, are invalid in cases in which the strong Jacobi bound is finite.

REMARK. In all that follows one can replace the set of all systems of $n$ differential polynomials in $n$ indeterminates by appropriate subsets (see [9]).

REMARK. One does not need characteristic 0 for the proofs of the theorems of this paper. However, the dimension conjecture is obviously false if the characteristic is positive. (The system $S=\left(y^{p}-a z^{p}\right)$, where $p$ is the characteristic and $a^{\prime} \neq 0$, has only the solution $y=z=0$.) In the spirit of the preceding remark, one might consider the dimension conjecture and Jacobi bound for systems satisfying an appropriate separability condition.

REMARK. The dimension conjecture is only known to hold for linear systems and in the case $k=1$. It holds also for partial differential equations in these cases.

REMARK. With minor modifications the results of this paper carry over to difference polynomials.

4. Definitions and known results. Let $A=\left(a_{i j}\right)$ be a $k \times n$ matrix. A transversal of $A$ is a subfamily of the $a_{i j}$ with no two entries in the same row or in the same column of $A$. A transversal with $\min (n, k)$ members is a diagonal. Let $\mathfrak{J}(A)=$ $\max _{D} \Sigma_{D} a_{i j}$, where $\Sigma_{D}$ is the sum over the diagonal $D$ of $A$.

Let $S=\left(P_{1}, \ldots, P_{k}\right)$ be a family of $k$ differential polynomials in $n$ differential indeterminates $y_{1}, \ldots, y_{n}$. Let $r_{i j}$ be the order of $P_{i}$ in $y_{j}$, if $y_{j}$ or any of its derivatives appears effectively in $P_{i}$, and let $r_{i j}=-\infty$ otherwise. Set $r_{i j}^{*}=\max \left(r_{i j}, 0\right)$. Let $R=\left(r_{i j}\right), R^{*}=\left(r_{i j}^{*}\right)$. We call $R$ and $R^{*}$ the strong and weak order matrices of $S$ respectively.

The strong Jacobi number $\mathfrak{\Im}(S)$ of $S$ is defined by $\mathfrak{\Im}(S)=\mathfrak{\Im}(R)$, where $R$ is the strong order matrix of $S$ and the obvious conventions are to be used with $-\infty$. The weak Jacobi number $\mathfrak{J}^{*}(S)$ of $S$ is defined by $\mathfrak{J}^{*}(S)=\mathfrak{\Im}\left(R^{*}\right)$, where $R^{*}$ is the weak order matrix of $S$.

It is conjectured that these numbers are bounds for the order, at least if $k=n$. More precisely, we have the following two conjectures:

If $S$ is a system with $k=n$, and $M$ is a component of the manifold of $S$ with differential dimension 0 , then the order of $M$ does not exceed $\mathfrak{s}(S)$.

If $S$ is a system with $k=n$, and $M$ is a component of the manifold of $S$ with differential dimension 0 , then the order of $M$ does not exceed $\mathfrak{\Im}^{*}(S)$.

The Ritt number $\Re(S)$ is defined to be the sum of the column maxima of $R^{*}$ (and the definition extends in an obvious way to systems of infinitely many differential polynomials of bounded orders), and the dual Ritt number $\mathfrak{I} \Re(S)$ is the sum of the row maxima of $R^{*}$.

Let $S$ have coefficients in the field $K$ of characteristic 0 , and let $k=n$. It is known that $\mathfrak{\Im}(S)$ and $\Im^{*}(S)$ are bounds for the order of the manifold of $S$ if $S$ is linear [7, 4, p. 199] and are the best possible bounds based on the information in $R$ and $R^{*}$ respectively [6, p. 133]. Without linearity, but with $n \leqslant 2$, they are bounds for the order of each component of the manifold of $S$ of differential dimension 0 (see $[7,8, p .136])$. These results have been extended (except for being best possible) to 
partial differential systems by Tomasovic [9] even without the requirement $n=k$. Lando [6] has shown that $i^{*}(S)$ is a bound for the order of each component of the manifold of $S$ of differential dimension 0 provided all $r_{i j} \leqslant 1$.

$\Re(S)$ is known to be a bound for the order of the components of the manifold of $S$ of differential dimension 0 [8, p. 135]; and this is so without the assumption $n=k$ and, indeed, for infinite systems of bounded orders in the indeterminates. The work of B. Greenspan and of Lando summarized in [1] has provided improvements on the bound $\Re(S)$, but seems unlikely to have yielded bounds which are best possible for the information in $R$ or $R^{*}$.

\section{Theorem of König and Egerváry.}

THEOREM. Let $A$ be a $k \times n$ matrix whose entries are 0 's and 1's. Let the maximal number of 1's in any diagonal of $A$ be $J<\min (n, k)$. Then $A$ contains a submatrix of 0 's with $a$ rows and $b$ columns, $0<a, b ; a+b=n+k-J$.

This theorem may be obtained by an easy restatement of the result proved in [5, p. 240]; and it is evident at once from the standard form to which Barbara Lando [6, p. 129] reduces matrices of 0 's and l's.

\section{The main result.}

THEOREM. If the dimension conjecture is false for systems of differential polynomials with coefficients in some extension of $K$, then there exists a family $T$ of differential polynomials with coefficients in some extension of $K$ such that $T$ has as many polynomials as the number of differential indeterminates it involves, $\Im(T) \neq-\infty$, and the manifold of $T$ has a component of differential dimension 0 and order exceeding $i(T)$. [In short, if the dimension conjecture is false in extensions of $K$, then the strong $J a c o b i$ number, even when finite, is not a valid bound for the order in extensions of $K$.]

Proof. By the hypothesis there exists a system $S=\left(P_{1}, \ldots, P_{k}\right)$ of differential polynomials in $L\left\{y_{1}, \ldots, y_{n}\right\}, n>k, L$ an extension of $K$, such that some component $M$ of the manifold of $S$ has differential dimension 0 .

Let $S$ be chosen so that $k$ is minimal for all choices of $L$. Note that $k>1$, and so $n>2$. Let $R$ be the strong order matrix of $S$. It will be shown that $R$ has a diagonal none of whose entries is $-\infty$. If not, it follows from the theorem of König and Egerváry that $R$ has a submatrix $H$ with $a$ rows and $b$ columns, $0<a, b ; a+b>n$, all of whose entries are $-\infty$. Let the numbering be such that $H$ is in the lower right-hand corner of $R$. Let $c=k-a, d=n-b$. Note that $c \neq 0$, for otherwise all components of $S$ have positive differential dimension. Let $\left(a_{1}, \ldots, a_{n}\right)$ be a generic zero of $M$. Let $P_{i}^{*}$ result from $P_{i}, 1 \leqslant i \leqslant c$, by the substitution of $a_{1}, \ldots, a_{d}$ for $y_{1}, \ldots, y_{d}$. Let $S^{*}=\left(P_{1}^{*}, \ldots, P_{c}^{*}\right)$. Suppose $b<n$. Then $S^{*}$ is a system of $c$ differential polynomials in the $b$ indeterminates $y_{d+1}, \ldots, y_{n}$ with coefficients in an extension of $K$. Let $\left(b_{d+1}, \ldots, b_{n}\right)$ be a solution of $S^{*}$. Then $\left(a_{1}, \ldots, a_{d}, b_{d+1}, \ldots, b_{n}\right)$ is a solution of $S$. Hence, $S^{*}$ has a component of differential dimension 0 . But since $c<n-a<b$, this contradicts the minimality of $k$. If $b=n, P_{k}=0$, and we may at 
once replace $S$ by $\left(P_{1}, \ldots, P_{k-1}\right)$, obtaining a contradiction. These contradictions show that $R$ has a diagonal with only finite entries.

It follows from the preceding paragraph that we may choose a $k \times k$ submatrix $A$ of $R$ with $\Im(A)>-\infty$. Renumber so that $A$ consists of the first $k$ columns of $R$. We may assume that $M$ is of order exceeding $\mathfrak{\Im}(A)$. For if $y_{n}$ is replaced in $S$ by $y_{n}^{(t)}$, then $S$ goes over into a system $S^{\prime}$ with a component $M^{\prime}$ of differential dimension 0 and order $t+$ order of $M$; while all else remains unchanged (see Appendix).

We extend $S$ to a system $S_{1}$ by adjoining $n-k$ new indeterminates $w_{i}$ and $2(n-k)$ new polynomials $A_{i}, B_{i}, k+1 \leqslant i \leqslant n$; where for each such $i, A_{i}=B_{i}=$ $w_{1}-y_{i}$. The system $S_{1}$ has as many polynomials as indeterminates.

Let $R_{1}$ be the strong order matrix for $S_{1}$. Then $R$ and $A$ are submatrices of $R_{1}$. For any $i, k+1 \leqslant i \leqslant n$, the row of $R_{1}$ which corresponds to either $A_{i}$ or $B_{i}$ has all its entries $-\infty$, except in the columns which correspond to the $y_{i}, w_{i}, k+1 \leqslant i \leqslant n$, in which columns it has 0 entries. Hence, a diagonal $D$ of $R_{1}$ which is free of the entry $-\infty$ must have the entry 0 in all rows corresponding to an $A_{i}$ or $B_{i}$. The diagonal $D$ consists therefore of a diagonal of the submatrix $A$ free of the entry $-\infty$, and of entries 0 from the rows corresponding to the $A_{i}$ and $B_{i}$. Conversely, every diagonal of $A$ free of the entry $-\infty$ extends to a diagonal of $R_{1}$ free of the entry $-\infty$ by adjoining 0 entries. It follows that is $\left(S_{1}\right)=i\left(R_{1}\right)=\Im(A)$. Since the manifold of $S_{1}$ evidently has a component $M_{1}$ of the same order as $M$, and the order of $M$ exceeds is $(A)$, we may choose $T=S_{1}$.

7. The weak Jacobi bound. Let $S=\left(P_{1}, \ldots, P_{n}\right)$ be a system of polynomials in $K\left\{y_{1}, \ldots, y_{n}\right\}$ such that $-\infty<\Im(S)<\Im^{*}(S)$; and suppose also that the manifold of $S$ has a component $M$ of differential dimension 0 and order exceeding $\Im(S)$. Let $R$ be the strong order matrix of $S$, and $R^{*}$ the weak order matrix.

Let $S_{1}$ be formed from $S$ by replacing each $y_{i}^{(K)}$ in $P_{j}$ by $y_{i}^{(K+1)}, 1 \leqslant i, j \leqslant n$, $K=0,1, \ldots$ Let $R_{1}, R_{1}^{*}$ be respectively the strong and weak order matrices of $S_{1}$.

The entries of $R_{1}^{*}$ exceed the corresponding entries of $R^{*}$ by 1 , except that those 0 entries in $R^{*}$ which correspond to the entries $-\infty$ in $R$ remain as 0 in $R_{1}^{*}$. Because is $(S)<\Im^{*}(S)$, the sum $\mathfrak{\Im}^{*}(S)$ is obtained only from diagonals of $R^{*}$ which correspond to diagonals of $R$ with at least one entry $-\infty$. These diagonals of $R^{*}$ give rise to diagonals of $R_{1}^{*}$ with sum at most $\mathfrak{\Im}^{*}(S)+n-1$. All other diagonals of $R$ have entries with sum less than $\mathfrak{S}^{*}(S)$, and therefore correspond to diagonals of $R_{1}$ whose entries have sum less than $\mathfrak{J}^{*}(S)+n$. It follows that $\mathfrak{\Im}^{*}\left(S_{1}\right)=\mathfrak{\Im}\left(R_{1}^{*}\right)<$ $\mathfrak{\Im}^{*}(S)+n$. On the other hand $\mathfrak{\Im}(S)$ is the sum of the entries in a diagonal of $R$ free of the entry $-\infty$; and this diagonal gives rise to a diagonal of $R_{1}$ whose entries have sum $\mathfrak{s}(S)+n$, which is clearly the maximal sum for diagonals of $R_{1}$. Hence, $\mathfrak{J}\left(S_{1}\right)=\mathfrak{J}\left(R_{1}\right)=\mathfrak{J}(S)+n$. It follows that $0 \leqslant \mathfrak{J}^{*}\left(S_{1}\right)-\mathfrak{J}\left(S_{1}\right)<\mathfrak{J}(S)-\mathfrak{J}(S)$.

To the component $M$ of the manifold of $S$ there corresponds a component $M_{1}$ of the manifold of $S_{1}$ of order $n+$ order of $M$ (see Appendix). After a sufficient number $h$ of repetitions of the above procedure one obtains a system $S_{h}$ such that $\mathfrak{\Im}^{*}\left(S_{h}\right)=\mathfrak{\Im}\left(S_{h}\right)=\mathfrak{\Im}(S)+n h$. The manifold of $S_{h}$ has a component $M_{h}$ of order $n h+\operatorname{order} M$. Hence, the order of $M_{h}$ exceeds $\mathfrak{J}(S)+n h=\mathfrak{J}^{*}\left(S_{h}\right)$. We have thus obtained the following corollary to the theorem of the preceding section. 
COROLlARY 1. Let $K$ be an ordinary differential field. If the dimension conjecture is false for systems of differential polynomials with coefficients in some extension of $K$, then the weak Jacobi number is not a bound for the order of components of systems with finite strong Jacobi number and coefficients in some extension of $K$.

A similar result can be proved for the dual Ritt number. Let $S=\left(P_{1}, \ldots, P_{n}\right)$ denote a family of differential polynomials in $K\left\{y_{1}, \ldots, y_{n}\right\}$ such that $\mathfrak{\Im}(S)$ is finite and $S$ has a component $M$ of differential dimension 0 and order exceeding $\mathfrak{J}^{*}(S)$. Let $R^{*}$ be the weak order matrix for $S$. By a result well known in connection with the solution of the assignment problem of integer programming, but actually due to Jacobi [2], there exist nonnegative integers $d_{1}, \ldots, d_{n}$ such that the matrix $R^{\prime}$ obtained by adding $d_{i}$ to each entry in the $i$ th column of $R^{*}, 1 \leqslant i \leqslant n$, has a diagonal consisting of row maxima. Then $\mathfrak{D} \Re\left(R^{\prime}\right)=\mathfrak{J}^{*}\left(R^{\prime}\right)$. It follows as in the earlier discussions that the dual Ritt number is not a bound for the orders of the components of differential dimension 0 of the system $S^{\prime}$ obtained from $S$ by replacing $y_{i}$ by $y_{i}^{\left(d_{1}\right)}, 1 \leqslant i \leqslant n$. This proves the following corollary.

COROLlARY 2. Let $K$ be an ordinary differential field. If the dimension conjecture is false for systems of differential polynomials with coefficients in extensions of $K$, then the dual Ritt number is not a bound for the order of components of systems with finite strong Jacobi number and coefficients in some extension of $K$.

REMARK. It is not evident how one could apply the method used to prove Corollary 2 to obtain a similar result for the Ritt number (which would amount to a proof of the dimension conjecture and the Jacobi bounds, since the Ritt number is a valid bound). For to do so one would have to modify a system $S$ not by replacing the indeterminates by their derivatives of appropriate orders, but by replacing the polynomials of the system by their derivatives of appropriate orders. The effect of such a replacement on the order of the system is not apparent.

Appendix. The following lemma justifies the substitutions used in the preceding proofs.

LEMMA. Let $K$ be an ordinary differential field. Let the manifold of a system $S$ of differential polynomials of $K\left\{y_{1}, \ldots, y_{n}\right\}$ have a component $M$ of differential dimension 0 . Let $S^{\prime}$ be obtained from $S$ by replacing $y_{1}^{(K)}$ by $y_{1}^{(K+1)}, K=0,1, \ldots$, in all the polynomials of $S$. Then the manifold of $S^{\prime}$ has a component $M^{\prime}$ of order $1+$ order of $M$.

Proof. Let $\left(a_{1}, \ldots, a_{n}\right)$ be a generic zero of $M$. Let $b$ be a generic zero of the prime differential ideal $I=\left\{z^{\prime}-a_{1}\right\}$ in $K\left\langle a_{1}, \ldots, a_{n}\right\rangle\{z\}$. Then $\left(b, a_{2}, \ldots, a_{n}\right)$ is a solution of $S^{\prime}$, and

$$
\text { t.d. } \begin{aligned}
K\left\langle b ; a_{2}, \ldots, a_{n}\right\rangle / K & =1+\text { t.d. } K\left\langle a_{1}, \ldots, a_{n}\right\rangle / K \\
& =1+\operatorname{order} \text { of } M .
\end{aligned}
$$

Let $M^{\prime}$ be a component of $S^{\prime}$ admitting the solution $\left(b, a_{2}, \ldots, a_{n}\right)$. Let $\left(g_{1}, \ldots, g_{n}\right)$ be a generic zero of $M^{\prime}$. Then $\left(g_{1}^{\prime} ; g_{2}, \ldots, g_{n}\right)$ is a zero of $S$ specializing differentially over $K$ to $\left(a_{1}, \ldots, a_{n}\right)$. This specialization must be generic since $M$ is a component of 
the manifold of $S$. Now t.d. $K\left\langle g_{1}, \ldots, g_{n}\right\rangle / K \geqslant$ t.d. $K\left\langle b, a_{2}, \ldots, a_{n}\right\rangle / K=1+$ order of $M$. But, t.d. $K\left\langle g_{1}, \ldots, g_{n}\right\rangle / K \leqslant 1+$ t.d. $K\left\langle g_{1}^{\prime} ; g_{2}, \ldots, g_{n}\right\rangle / K=1+$ order of $M$; where the equality holds because $\left(g_{1}^{\prime} ; g_{2}, \ldots, g_{n}\right)$ is generic for $M$ over $K$. Thus, ord $M^{\prime}=$ t.d. $K\left\langle g_{1}, \ldots, g_{n}\right\rangle / K=1+$ order of $M$.

\section{REFERENCES}

1. R. M. Cohn, The Greenspan bound for the order of differential systems, Proc. Amer. Math. Soc. 79 (1980), 523-526.

2. C. G. J. Jacobi, De investigando ordine systematis aequationum differentialium vulgarium cujuscunque, Gesammelte Werke, Vol 5, Chelsea, New York, pp. 193-216.

3. I. Kaplansky, An introduction to differential algebra, Hermann, Paris, 1957.

4. E. R. Kolchin, Differential algebra and algebraic groups, Academic Press, New York and London, 1973.

5. D. König, Theorie der Endlichen und Unendlichen Graphen, Chelsea, New York, 1950.

6. B. A. Lando, Jacobi's bound for the order of systems of first order differential equations. Trans. Amer. Math. Soc. 152 (1970), 119-135.

7. J. F. Ritt, Jacobi's problem on the order of a system of differential equations, Ann. of Math. (2) 36 (1935), 303-312.

8. _ Differential algebra, Amer. Math. Soc. Colloq. Publ., vol 33, Amer. Math. Soc., Providence, R. I., 1950.

9. S. J. Tomasovic, Jr., A generalized Jacobi conjecture for arbitrary systems of algebraic differential equations, Dissertation, Columbia University, 1976.

Department of Mathematics, Rutgers University, New Brunswick, New Jersey 08903 